SISSA REF 16/2002/FM

DFTT $9 / 2002$

\title{
On the fermion-boson correspondence for correlation functions of disorder operators
}

\author{
G. Delfino ${ }^{a}$, P. Grinza $^{b}$ and G. Mussardo ${ }^{a}$ \\ a SISSA and INFN, via Beirut 2-4, 34014 Trieste, Italy \\ ${ }^{b}$ Dipartimento di Fisica and INFN, \\ Università degli Studi di Torino, via Giuria 1, 10125 Torino, Italy
}

\begin{abstract}
When a quantum field theory possesses topological excitations in a phase with spontaneously broken symmetry, these are created by operators which are non-local with respect to the order parameter. Due to non-locality, such disorder operators have non-trivial correlation functions even in free massive theories. In two dimensions, these correlators can be expressed exactly in terms of solutions of non-linear differential equations. The correlation functions of the one-parameter family of non-local operators in the free charged bosonic and fermionic models are the inverse of each other. We point out a simple derivation of this correspondence within the form factor approach.
\end{abstract}


1. The existence of excitations owing their stability to topological reasons is a well known non-perturbative feature of quantum field theory. In a phase with spontaneously broken symmetry, field configurations may exist which interpolate at spatial infinity among different vacua of the theory and possess a topologic charge. In the quantum theory, these 'extended' objects (solitons, vortices,..) must be created by the action on the vacuum of operators which are non-local with respect to the order parameter. Since the operator content of the theory is expected to be the same on the two sides of the phase transition, such disorder operators can be identified also in the unbroken phase, where the topologic excitations are absent and they acquire non-zero vacuum expectation values.

In two space-time dimensions, only discrete symmetries can be broken spontaneously. Whenever this is the case, the broken phase possesses 'kinks' interpolating between two different vacua. As a concrete example, consider the (euclidean) action

$$
\mathcal{A}=\int d^{2} x\left[\partial_{\mu} \phi^{*} \partial^{\mu} \phi+m^{2} \phi^{*} \phi+W\left(\phi^{*}, \phi\right)\right]
$$

where the potential $W$ is invariant under the $Z_{N}$ transformation $\phi \rightarrow e^{2 i \pi / N} \phi, \phi^{*} \rightarrow$ $e^{-2 i \pi / N} \phi^{*}$. Denote $\left|0_{j}\right\rangle, j=0,1, \ldots, N-1$, the $N$ vacuua of the broken phase. Then we call $\mu_{k}(x), k=1, \ldots, N-1$, the disorder operators which create the excitations interpolating between the vacua $\left|0_{j}\right\rangle$ and $\left|0_{j+k(\bmod N)}\right\rangle$. The operators $\mu_{k}(x)$ carry $k$ units of topological charge and satisfy the conjugation relation $\mu_{k}^{*}(x)=\mu_{N-k}(x)$. The mutual non-locality between the order and disorder operators manifests itself in a phase factor arising when they are taken around each other in the euclidean plane $\left(z=x_{1}+i x_{2}\right.$, $\left.\bar{z}=x_{1}-i x_{2}\right):$

$$
\begin{aligned}
\phi\left(z e^{2 i \pi}, \bar{z} e^{-2 i \pi}\right) \mu_{k}(0,0) & =e^{2 i \pi k / N} \phi(z, \bar{z}) \mu_{k}(0,0) \\
\phi^{*}\left(z e^{2 i \pi}, \bar{z} e^{-2 i \pi}\right) \mu_{k}(0,0) & =e^{-2 i \pi k / N} \phi^{*}(z, \bar{z}) \mu_{k}(0,0) .
\end{aligned}
$$

These analytic continuations apply inside correlation functions and imply that the latter are not single valued. They hold along any $Z_{N}$-invariant renormalisation group trajectory flowing out of the phase transition point and characterise the operators $\mu_{k}(x)$ beyond their role of kink creation operators in the broken phase.

The theory (11) with $W=0$ is a particularly simple example of such a trajectory. Even in this case the correlation functions of disorder operators are non-trivial due to the non-locality with respect to the boson. One can hope, however, to compute them exactly exploiting the free particle basis, in analogy with what happens for the Ising field theory without magnetic field, where the spin correlators can be computed exactly due to the underlying free fermion theory [1] 
The correlation functions of non-local operators in two-dimensional free massive theories were extensively studied from the point of view of the deformation theory of differential equations in Ref. [2] and a series of related papers. Several works which include Refs. [3, 4, 5, 6, 7] have been devoted afterwards to dealing with this problem through more direct and general methods of quantum field theory. In particular, the attention focused on the form factor approach in which the correlators are expressed as sums over multiparticle asymptotic states. While these spectral series can be written down explicitely for any integrable quantum field theory, the free case is up to now the only one in which they can be resummed. The use of this approach to express the correlators through solutions of non-linear differential equations was illustrated in [6] and [7] for the neutral and charged fermionic cases, respectively.

In this note we point out that the form factor approach allows for a simple unified treatment of the bosonic and fermionic cases showing the correspondence巴 [2]

$$
\left\langle\tilde{\mu}_{j}(x) \tilde{\mu}_{k}(0)\right\rangle=\frac{1}{\left\langle\tilde{V}_{j / N}(x) \tilde{V}_{k / N}(0)\right\rangle},
$$

where the correlators on the l.h.s. are computed in the theory (1) with $W=0$, and those on the r.h.s. refer to the operators $V_{\alpha}(x)=\exp [i \sqrt{4 \pi} \alpha \varphi(x)]$ in the sine-Gordon theory

$$
\mathcal{A}_{s G}=\int d^{2} x\left[\frac{1}{2} \partial_{\nu} \varphi \partial^{\nu} \varphi-\mu \cos \beta \varphi\right],
$$

with $\beta=\sqrt{4 \pi}$ and $\mu$ a suitably chosen mass scale.

We recall that the theory (5) with $\beta=\sqrt{4 \pi}$ is in fact a free fermionic theory $[\mathbb{8}]$. For generic values of $\beta$ the elementary excitations are the solitons and antisolitons interpolating between adjacent vacua of the periodic potential. Their interaction is attractive as long as $\beta<\sqrt{4 \pi}$ and they form topologically neutral bound states, the lightest one being the particle interpolated by the bosonic field in the action (5). These neutral particles are absent from the spectrum of asymptotic states in the repulsive region $\beta>\sqrt{4 \pi}$, and at the point $\beta=\sqrt{4 \pi}$ where the solitons behave as free Dirac fermions.

Since the solitons are non-local in terms of the field $\varphi(x)$, the evaluation of both sides of Eq. (47) amounts to computing correlation functions of operators which are non-local with respect to non-interacting particles, bosons for the l.h.s. and fermions for the r.h.s. We will show how the different statistics produces the inversion in Eq. (4).

2. Consider a theory of free, charge conjugated particles $A$ and $\bar{A}$ with mass $m$, and denote by $\Phi_{\alpha}(x)$ a scalar operator exhibiting (in the sense of (2), (3)) ) a non-locality phase

\footnotetext{
${ }^{1}$ For a given operator $\Phi(x)$, the notation $\tilde{\Phi}(x) \equiv \Phi(x) /\langle\Phi\rangle$ will be used throughout this note.
} 
$e^{2 i \pi \alpha}\left(e^{-2 i \pi \alpha}\right)$ with respect to (the field which interpolates) the particle $A(\bar{A})$. Acting on the vacuum state $|0\rangle$, such an operator produces neutral states consisting of pairs $A(\theta) \bar{A}(\beta)$. We use rapidity variables to parameterise the energy-momentum of a particle as $(e, p)=(m \cosh \theta, m \sinh \theta)$. The equations satisfied by the form factors

$$
f_{n}^{\alpha}\left(\theta_{1}, \ldots, \theta_{n}, \beta_{1}, \ldots, \beta_{n}\right)=\left\langle 0\left|\tilde{\Phi}_{\alpha}(0)\right| A\left(\theta_{1}\right), \ldots, A\left(\theta_{n}\right), \bar{A}\left(\beta_{1}\right), \ldots, \bar{A}\left(\beta_{n}\right)\right\rangle
$$

are a particular case of those holding for generic integrable theories (see e.g. [5])

$$
\begin{aligned}
f_{n}^{\alpha}\left(\theta_{1}, \ldots, \theta_{i}, \theta_{i+1}, \ldots, \theta_{n}, \beta_{1}, \ldots, \beta_{n}\right) & =S f_{n}^{\alpha}\left(\theta_{1}, \ldots, \theta_{i+1}, \theta_{i}, \ldots, \theta_{n}, \beta_{1}, \ldots, \beta_{n}\right), \\
f_{n}^{\alpha}\left(\theta_{1}+2 i \pi, \theta_{2}, \ldots, \theta_{n}, \beta_{1}, \ldots, \beta_{n}\right) & =S e^{2 i \pi \alpha} f_{n}^{\alpha}\left(\theta_{1}, \ldots, \theta_{n}, \beta_{1}, \ldots, \beta_{n}\right), \\
\operatorname{Res}_{\theta_{1}-\beta_{1}=i \pi} f_{n}^{\alpha}\left(\theta_{1}, \ldots, \theta_{n}, \beta_{1}, \ldots, \beta_{n}\right) & =i S^{n-1}\left(1-e^{2 i \pi \alpha}\right) f_{n-1}^{\alpha}\left(\theta_{2}, . ., \theta_{n}, \beta_{2}, . ., \beta_{n}\right),
\end{aligned}
$$

where

$$
S=\left\{\begin{array}{cl}
1 & \text { for free bosons } \\
-1 & \text { for free fermions }
\end{array}\right.
$$

It is easy to check that the solution of the above system of equations is given by

$$
f_{n}^{\alpha}\left(\theta_{1}, \ldots, \theta_{n}, \beta_{1}, \ldots, \beta_{n}\right)=S^{n(n+2) / 2}(-\sin \pi \alpha)^{n} e^{\left(\alpha-\frac{1}{2} \delta_{S, 1}\right) \sum_{i=1}^{n}\left(\theta_{i}-\beta_{i}\right)}\left|A_{n}\right|_{(S)}
$$

where $A_{n}$ is a $n \times n$ matrix $\left(A_{0} \equiv 1\right)$ with entries

$$
A_{i j}=\frac{1}{\cosh \frac{\theta_{i}-\beta_{j}}{2}}
$$

and $\left|A_{n}\right|_{(S)}$ denotes the permanent? of $A_{n}$ for $S=1$ and the determinant of $A_{n}$ for $S=-1$. In fact, eq. (7) (and the analogous equation for the permutation of two $\beta$ variables) immediately follows from the property of $|A|_{(S)}$ under exchange of two rows or columns. Concerning eq. (8), the minus sign produced by $|A|_{(S)}$ when $\theta_{1} \rightarrow \theta_{1}+2 i \pi$ is the one needed in the fermionic case, while it is canceled by the action of the Kroneker delta when $S=1$. Finally, eq. (9) follows from the fact that taking the residue at $\theta_{1}-\beta_{1}=i \pi$ amounts to deleting the first row and the first column of $A_{n}$, which produces $\left|A_{n-1}\right|_{(S)}$.

The two-point correlators are given by

$$
G_{\alpha, \alpha^{\prime}}^{(S)}(t)=\left\langle\tilde{\Phi}_{\alpha}(x) \tilde{\Phi}_{\alpha^{\prime}}(0)\right\rangle=\sum_{n=0}^{\infty} \frac{1}{(n !)^{2}(2 \pi)^{2 n}} \int d \theta_{1} \ldots d \theta_{n} d \beta_{1} \ldots d \beta_{n} g_{n}^{\left(\alpha, \alpha^{\prime}\right)}\left(t \mid \theta_{1}, \ldots, \beta_{n}\right),
$$

\footnotetext{
${ }^{2}$ The permanent of a matrix differs from the determinant by the omission of the alternating sign factors $(-1)^{i+j}$.
} 
where

$$
\begin{gathered}
g_{n}^{\left(\alpha, \alpha^{\prime}\right)}\left(t \mid \theta_{1}, \ldots, \beta_{n}\right)=f_{n}^{\alpha}\left(\theta_{1}, \ldots, \beta_{n}\right) f_{n}^{\alpha^{\prime}}\left(\beta_{n}, \ldots, \theta_{1}\right) e^{-t e_{n}} \\
=\left(S \sin \pi \alpha \sin \pi \alpha^{\prime}\right)^{n} e^{\left(\alpha-\alpha^{\prime}\right) \sum_{i=1}^{n}\left(\theta_{i}-\beta_{i}\right)}\left|A_{n}\right|_{(S)}^{2} e^{-t e_{n}}, \\
t=m|x| \quad, \quad e_{n}=\sum_{k=1}^{n}\left(\cosh \theta_{k}+\cosh \beta_{k}\right) .
\end{gathered}
$$

Analogously to the fermionic case [7], we define a new $n \times n$ matrix $M_{n}$ with entries

$$
\begin{gathered}
M_{i j} \equiv M\left(\theta_{i}, \beta_{j}\right)=\left(\sin \pi \alpha \sin \pi \alpha^{\prime}\right)^{1 / 2} e^{-\frac{t}{2} \cosh \theta_{i}} \frac{h\left(\theta_{i}\right) h^{-1}\left(\beta_{j}\right)}{\cosh \frac{\theta_{i}-\beta_{j}}{2}} e^{-\frac{t}{2} \cosh \beta_{j}}, \\
h(\theta)=e^{\left(\alpha-\alpha^{\prime}\right) \theta / 2},
\end{gathered}
$$

and rewrite eq. (14) as

$$
g_{n}^{\left(\alpha, \alpha^{\prime}\right)}=S^{n}\left|M_{n}\right|_{(S)}^{2}=\left|\begin{array}{cc}
0 & M_{n} \\
M_{n}^{T} & 0
\end{array}\right|_{(S)}
$$

Finally we symmetrise with respect to the two sets of rapidities by introducing a charge index $\varepsilon$ which is 1 for a particle $A$ and -1 for $\bar{A}$, so that we express eq. (13) as

$$
G_{\alpha, \alpha^{\prime}}^{(S)}(t)=\sum_{L=0}^{\infty} \frac{1}{L !(2 \pi)^{L}} \sum_{\epsilon_{1} \ldots \epsilon_{L}} \int d \theta_{1} \ldots d \theta_{L}\left|K_{\epsilon_{i} \epsilon_{j}}\left(\theta_{i}, \theta_{j}\right)\right|_{(S)}
$$

where we have introduced the $L \times L$ matrices with entries

$$
\begin{aligned}
& K_{+-}(\theta, \beta)=M(\theta, \beta), \\
& K_{-+}(\theta, \beta)=M(\beta, \theta), \\
& K_{++}(\theta, \beta)=K_{--}(\theta, \beta)=0 .
\end{aligned}
$$

The last equation in (18) ensures that only the terms with $L=2 n$ occur in (17). Notice that the dependence on the statistics in (17) only reduces to taking the permanent or the determinant of the same matrix. According to the theory of Fredholm integral operators (see e.g. [9]) this leads to the result

$$
G_{\alpha, \alpha^{\prime}}^{(S)}(t)=\operatorname{Det}(1+2 \pi \mathbf{K})^{-S}
$$

so that the correlators of operators having the same non-locality phase in the free fermion and free boson theories are the inverse of each other. The result (4) follows from the fact that the operators $V_{\alpha}(x)$ have a non-locality phase $e^{2 i \pi \alpha}$ around the solitons at the 
sine-Gordon free fermion point (see e.g. [10]), so that $\mu_{j}(x)$ and $V_{j / N}(x)$ have the same non-locality with respect to the corresponding particles. Since $j$ runs between 1 and $N-1$, we are actually working with $0<\alpha=j / N<1$.

3. We have derived Eq. (4) by using the large distance expansion of the correlators. It is interesting to check this inversion relation between the two correlators in the short distance limit. Both in the bosonic and fermionic case the operators $\Phi_{\alpha}(x)$ satisfy the operator product expansion

$$
\left\langle\Phi_{\alpha}(x) \Phi_{\alpha^{\prime}}(0)\right\rangle \sim|x|^{-\Gamma_{\alpha, \alpha^{\prime}}^{(S)}}\left\langle\Phi_{\alpha+\alpha^{\prime}}\right\rangle+\ldots
$$

with

$$
\Gamma_{\alpha, \alpha^{\prime}}^{(S)}=X_{\alpha}^{(S)}+X_{\alpha^{\prime}}^{(S)}-X_{\alpha+\alpha^{\prime}}^{(S)}
$$

$X_{\alpha}^{(S)}$ being the scaling dimensions. In the bosonic case the index $\alpha+\alpha^{\prime}$ is taken modulo 1. The scaling dimensions can be computed through the formula [11]

$$
X_{\alpha}^{(S)}=-\frac{1}{2 \pi} \int d^{2} x\left\langle\Theta(x) \tilde{\Phi}_{\alpha}(0)\right\rangle_{\text {connected }}
$$

where $\Theta(x)$ is the trace of the energy-momentum tensor. Since the only non-zero form factor of this operator in the free theories is

$$
\langle 0|\Theta(0)| A(\theta) \bar{A}(\beta)\rangle=2 \pi m^{2}\left[-i \sinh \frac{\theta-\beta}{2}\right]^{\delta_{S,-1}},
$$

one easily finds

$$
X_{\alpha}^{(S)}= \begin{cases}\alpha(1-\alpha), & S=1 \\ \alpha^{2}, & S=-1\end{cases}
$$

in agreement with the conjugation properties $\mu_{j}^{*}=\mu_{N-j}$ and $V_{\alpha}^{*}=V_{-\alpha}$. These results coincide with those of conformal field theory with 'twist' fields (see [12, 13]). It follows

$$
\begin{gathered}
\Gamma_{\alpha, \alpha^{\prime}}^{(-)}=-2 \alpha \alpha^{\prime}, \\
\Gamma_{\alpha, \alpha^{\prime}}^{(+)}= \begin{cases}2 \alpha \alpha^{\prime}, & \alpha+\alpha^{\prime}<1 \\
2\left[\alpha \alpha^{\prime}+1-\left(\alpha+\alpha^{\prime}\right)\right], & \alpha+\alpha^{\prime}>1 .\end{cases}
\end{gathered}
$$

The agreement for $1<\alpha+\alpha^{\prime}<2$ is obtained by observing that in this range of $\alpha+\alpha^{\prime}$, the leading short distance term in the fermionic case is not the one in (20) but the first off-critical contribution

$$
\mu / 2 \int d^{2} y\left\langle V_{\alpha}(x) V_{\alpha}(0)\left[V_{1}(y)+V_{-1}(y)\right]\right\rangle_{\mu=0}
$$


which indeed behaves as $|x|^{2\left[\alpha \alpha^{\prime}+1-\left(\alpha+\alpha^{\prime}\right)\right]}$ when $x \rightarrow 0$.

In general, the two-point functions can be expressed as

$$
G_{\alpha, \alpha^{\prime}}^{(S)}(t)=e^{S \Upsilon_{\alpha, \alpha^{\prime}}(t)} .
$$

where $\Upsilon_{\alpha, \alpha^{\prime}}(t)$ is given by [2, []

$$
\Upsilon_{\alpha, \alpha^{\prime}}(t)=\frac{1}{2} \int_{t / 2}^{\infty} \rho d \rho\left[\left(\partial_{\rho} \chi\right)^{2}-4 \sinh ^{2} \chi-\frac{\left(\alpha-\alpha^{\prime}\right)^{2}}{\rho^{2}} \tanh \chi\right],
$$

with $\chi(\rho)$ satisfying the differential equation

$$
\partial_{\rho}^{2} \chi+\frac{1}{\rho} \partial_{\rho} \chi=2 \sinh 2 \chi+\frac{\left(\alpha-\alpha^{\prime}\right)^{2}}{\rho^{2}} \tanh \chi\left(1-\tanh ^{2} \chi\right) .
$$

The precise short distance behaviour for $\alpha+\alpha^{\prime}<1$ is

$$
\lim _{t \rightarrow 0} G_{\alpha, \alpha^{\prime}}^{(S)}(t)=\left(C_{\alpha, \alpha^{\prime}} t^{2 \alpha \alpha^{\prime}}\right)^{-S},
$$

with an amplitude that can be deduced from the work of Ref. [14] on vacuum expectation values in the sine-Gordon model:

$$
C_{\alpha, \alpha^{\prime}}=2^{-2 \alpha \alpha^{\prime}} \exp \left\{2 \int_{0}^{\infty} \frac{d t}{t}\left[\frac{\sinh \alpha t \cosh \left(\alpha+\alpha^{\prime}\right) t \sinh \alpha^{\prime} t}{\sinh ^{2} t}-\alpha \alpha^{\prime} e^{-2 t}\right]\right\} .
$$

4. The case of $Z_{2}$ symmetry is somehow special. Since a broken phase with two degenerate vacua can be realised in terms of a neutral boson, a disorder operator with non-locality factor -1 in present also in the theory of a neutral bosonic free particle. It is easy to check that, for $S=1$ and $\alpha=1 / 2$, the form factors (111) imply the factorisation

$$
\mu_{1}=\mu_{(1)} \times \mu_{(2)}, \quad N=2
$$

where $\mu_{(j)}$ is the disorder operator with scaling dimension $X_{1 / 2}^{(+)} / 2=1 / 8$ associated to the neutral boson $A_{j}$ entering the decomposition $A=\left(A_{1}+i A_{2}\right) / \sqrt{2}, \bar{A}=\left(A_{1}-i A_{2}\right) / \sqrt{2}$.

The fermion-boson correspondence observed above for charged particles has an analogue in the neutral case, and the correlation function

$$
G(t)=\left\langle\tilde{\mu}_{(j)}(x) \tilde{\mu}_{(j)}(0)\right\rangle=\left[G_{1 / 2,1 / 2}^{(+)}(t)\right]^{1 / 2}
$$

can be related to correlators computed in the theory of a free neutral fermion, i.e. in the Ising field theory without magnetic field. To see this, let us recall that the correlators of 
the spin and disorder operators in the unbroken phase of the scaling Ising model can be written as [1]

$$
\tau_{ \pm}(t) \equiv\langle\tilde{\mu}(x) \tilde{\mu}(0)\rangle \pm\langle\sigma(x) \sigma(0)\rangle=\exp \left[ \pm \frac{1}{2} \chi(t / 2)-\frac{1}{2} \Upsilon_{1 / 2,1 / 2}(t)\right],
$$

where the functions $\chi$ and $\Upsilon$ are those of Eqs. (29), (30). Hence, it follows from (28) and (34) that

$$
G(t)=\left[\tau_{+}(t) \tau_{-}(t)\right]^{-1 / 2}
$$

Concerning the short distance behaviour of this correlator, the power law (31) acquires in this case a logarithmic correction due to the 'resonance' with the leading off-critical contribution (27). Such a contribution to the correlator $\left\langle\tilde{V}_{\alpha}(x) \tilde{V}_{\alpha}(0)\right\rangle$ behaves as $-C_{\alpha, \alpha} t^{1 / 2}[1+$ $(2-4 \alpha) \ln t]$ in the limit $\alpha \rightarrow 1 / 2$, so that

$$
\lim _{t \rightarrow 0} G_{1 / 2,1 / 2}^{(-)}(t)=\lim _{t \rightarrow 0} G^{-2}(t)=\mathcal{B} t^{1 / 2} \ln (1 / t),
$$

with

$$
\mathcal{B}=-4 \operatorname{Res}_{\alpha=1 / 2} C_{\alpha, \alpha}=0.588353 . .
$$

We checked that this amplitude coincides with that of the product $\tau_{+}(t) \tau_{-}(t)$ in the Ising model.

5. The free bosonic and fermionic theories discussed above can also be regarded as describing phases of spontaneously broken $Z_{N}$ symmetry. In this dual vision, the excitations are free kinks $\left|K_{j, j \pm 1}(\theta)\right\rangle$ interpolating between two adjacent vacua $\left|0_{j}\right\rangle$ and $\left|0_{j \pm 1}\right\rangle$ (the indices are taken modulo $N$ ). We still denote by $\Phi_{k / N}, k=0,1, \ldots, N-1$, the operators we are interested in. They correspond to the exponential operators $V_{k / N}$ in the fermionic cases, and to the operators $\sigma_{k}$, dual to the disorder operators $\mu_{k}$, in the bosonic case. These operators create multikink excitations with zero topologic charge, i.e. starting and ending in the same vacuum state. Form factors on kink states were discussed in [15]. Consider for the sake of simplicity the two-kink matrix elements

$$
F_{j, k}^{ \pm}\left(\theta_{1}-\theta_{2}\right) \equiv\left\langle 0_{j}\left|\Phi_{k / N}(0)\right| K_{j, j \pm 1}\left(\theta_{1}\right) K_{j \pm 1, j}\left(\theta_{2}\right)\right\rangle
$$

satisfying the equations

$$
\begin{aligned}
F_{j, k}^{ \pm}(\theta) & =S F_{j, k}^{\mp}(-\theta) \\
F_{j, k}^{ \pm}(\theta+2 i \pi) & =F_{j \pm 1, k}^{\mp}(-\theta), \\
\operatorname{Res}_{\theta=i \pi} F_{j, k}^{ \pm}(\theta) & =i\left[\left\langle 0_{j}\left|\Phi_{k / N}\right| 0_{j}\right\rangle-\left\langle 0_{j \pm 1}\left|\Phi_{k / N}\right| 0_{j \pm 1}\right\rangle\right] .
\end{aligned}
$$

\footnotetext{
${ }^{3}$ In this case the $\mathrm{N}$ degenerate vacua can be identified with those of the periodic potential in (5) identified modulo N.
} 
Since the generator $\Omega$ of $Z_{N}$ transformations $\left(\Omega^{N}=1\right)$ acts on states and operators as $\left(\omega \equiv e^{2 i \pi / N}\right)$

$$
\begin{aligned}
\Omega\left|K_{j, j+1}\left(\theta_{1}\right) K_{j+1, j+2}\left(\theta_{2}\right) \ldots\right\rangle & =\left|K_{j+1, j+2}\left(\theta_{1}\right) K_{j+2, j+3}\left(\theta_{2}\right) \ldots\right\rangle \\
\Omega^{-1} \Phi_{k / N}(x) \Omega & =\omega^{k} \Phi_{k / N}(x)
\end{aligned}
$$

the above form factor equations can be rewritten as

$$
\begin{aligned}
F_{j, k}^{ \pm}(\theta+2 i \pi) & =S \omega^{ \pm k} F_{j, k}^{ \pm}(\theta) \\
\operatorname{Res}_{\theta=i \pi} F_{j, k}^{ \pm}(\theta) & =i\left(1-\omega^{ \pm k}\right)\left\langle 0_{j}\left|\Phi_{k / N}\right| 0_{j}\right\rangle .
\end{aligned}
$$

Once the identifications $A \longleftrightarrow K_{i, i+1}, \bar{A} \longleftrightarrow K_{i, i-1}$ are made, these relations are equivalent to eqs. (8), (9) with $n=1$. The correspondence is easily extended to all values of $n$ and leads to the same form factors and correlation functions discussed for the unbroken phase.

The introduction of a 'magnetic field' pointing in the $k$ direction and breaking explicitely the $Z_{N}$ symmetry corresponds to adding to the free action a term

$$
h \int d^{2} x \Psi_{k}(x)
$$

where

$$
\Psi_{k}(x)=\frac{1}{N} \sum_{l=0}^{N-1} \omega^{-k l} \Phi_{l / N}(x) .
$$

The first order corrections to the energy density $\varepsilon_{j}$ of the vacuum state $\left|0_{j}\right\rangle$ and to the mass $m_{j, j \pm 1}$ of the kink $K_{j, j \pm 1}$ are 16

$$
\begin{aligned}
& \delta \varepsilon_{j} \sim h\left\langle 0_{j}\left|\Psi_{k}\right| 0_{j}\right\rangle=h v \delta_{j, k} \\
& \delta m_{j, j \pm 1}^{2} \sim h\left\langle 0_{j}\left|\Psi_{k}(0)\right| K_{j, j \pm 1}(i \pi) K_{j \pm 1, j}(0)\right\rangle .
\end{aligned}
$$

It follows from eq. (42) that

$$
\operatorname{Res}_{\theta=i \pi}\left\langle 0_{j}\left|\Psi_{k}(0)\right| K_{j, j \pm 1}(\theta) K_{j \pm 1, j}(0)\right\rangle=\frac{i v}{N}\left(\delta_{j, k}-\delta_{j, k \mp 1}\right)
$$

implying that the correction to the mass of the kinks interpolating between the vacua $\left|0_{k}\right\rangle$ and $\left|0_{k \pm 1}\right\rangle$ is infinite. This divergence simply reflects the fact that these kinks become unstable because the magnetic field removes the degeneracy of the vacuum $\left|0_{k}\right\rangle$ with the two adjacent vacua. These kinks are then confined in pairs $K_{k, k \pm 1} K_{k \pm 1, k}$ and the confinement gives rise to a string of bound states with zero topologic charge, as discussed in 17 . 


\section{References}

[1] T.T. Wu, B.M. McCoy, C.A. Tracy and E. Barouch, Phys. Rev. B 13 (1976) 316.

[2] M. Sato, T. Miwa and M. Jimbo, Publ. RIMS, Kyoto Univ., 15 (1979) 871.

[3] B. Berg, M. Karowski and P. Weisz, Phys. Rev. D 19 (1979) 2477.

[4] E.C. Marino, B. Schroer and J.A. Swieca, Nucl. Phys. B 200 (1982) 473.

[5] V.P. Yurov and Al.B. Zamolodchikov, Int. J. Mod. Phys. A6 (1991) 3419.

[6] O. Babelon and D. Bernard, Phys. Lett. B288 (1992) 113.

[7] D. Bernard and A. LeClair, Nucl. Phys. B 426 (1994) 534; Erratum-ibid. B 498 (1994) 619; hep-th/9402144.

[8] S. Coleman, Phys. Rev. D 11 (1975) 2088.

[9] J. Schwinger, Phys. Rev. 92 (1953) 1283.

[10] G. Delfino, J. Phys. A 34 (2001) L311.

[11] G. Delfino, P. Simonetti and J. Cardy, Phys. Lett. B 387 (1996) 327.

[12] L. Dixon, D. Friedan, E. Martinec and S. Shenker, Nucl. Phys. B 282 (1987) 13.

[13] Al.B. Zamolodchikov, Nucl. Phys. B 285 (1987) 481.

[14] S. Lukyanov and A.B. Zamolodchikov, Nucl. Phys. B 493 (1997) 571.

[15] G. Delfino and J.L. Cardy, Nucl. Phys. B 519 (1998) 551.

[16] G. Delfino, G. Mussardo and P. Simonetti, Nucl. Phys. B 473 (1996) 469.

[17] G. Delfino and G. Mussardo, Nucl. Phys. B 516 (1998) 675. 